\title{
Rare Tumors of Ovary: Case Report and Literature Review*
}

\author{
El Fatemi Hinde ${ }^{1 \#}$, Hammas Nawal ${ }^{1}$, Saadi Hannane ${ }^{2}$, Brahmi Sami $^{3}$, Amarti Afaf ${ }^{1}$, \\ Bannani Abdelaziz ${ }^{2}$, Mesbahi Omar ${ }^{3}$
}

${ }^{1}$ Department of Pathology, Hassan II Teaching Hospital, Fez, Morocco; ${ }^{2}$ Department of Gynecology, Hassan II Teaching Hospital, Fez, Morocco; ${ }^{3}$ Department of Oncology, Hassan II Teaching Hospital, Fez, Morocco.

Email: \{ Hinde0012, abdbannani, amartia\}@hotmail.com, \{mi-mika, h.saadi, b.sami\}@live.fr, omesbahi@yahoo.fr

Received May $15^{\text {th }}$, 2012; revised June $10^{\text {th }}$, 2012; accepted June $17^{\text {th }}, 2012$

\begin{abstract}
Introduction: Large cell neuroendocrine carcinoma (LCNEC) or non small cell neuroendocrine carcinoma the ovary is a rare entity and is frequently associated with ovarian surface epithelial tumors. However, its association with serous carcinoma is rarer, in our knowledge tree cases has been described in international literature and the first case in Moroccan literature. Case Report: A 54-year-old woman presented with a pelvic mass measuring $15 \mathrm{~cm}$ in diameter. She underwent an exploratory laparotomy with resection of the pelvic mass. Diffuse and nodular intra-abdominal disseminations were observed. Immunohistochemistry stain confirmed the diagnosis of large cell neuroendocrine carcinoma with serous carcinoma. The patient received three courses of carboplatin and paclitaxel and she's still alivewith a decline of 6 months. Its clinicopathologic association is discussed and the literature is reviewed. Conclusion: In summary, ovarian LCNEC is an aggressive tumor with a tendency to present at advanced stage and cause death within a mean of 17 months after diagnosis; however, some patients, particularly those with stage I disease and/or those who have received platinum-based therapy, may have a more favorable prognosis.
\end{abstract}

Keywords: Ovary; Neuroendocrine Carcinoma; Non-Small Cell Type; Serous Carcinoma; Immunohistochemistry

\section{Introduction}

Large cell neuroendocrine carcinoma (LCNEC) of the ovary is a rare tumor and is now included in the World Health Organization tumor classification [1]. Its prognosis is generally very poor even when the diagnosis is made at an early stage. A few cases of primary ovarian LCNECs have been reported. The majority of the tumors are associated with other epithelial neoplasms, among which only one case of serous carcinoma has been recently described [2]. Here we present another case of LCNEC of the ovary associated with serous carcinoma and is the first described to our knowledge in Moroccan literature.

\section{Case Report}

A 54-year-old G4P4 woman presented with abdominal distension for 5 months. Her past medical history was remarkable only for hypertension. Physical examination was remarkable for ascites, bilateral firm fixed mass in the pelvis. A chest radiograph showed no metastatic disease. Computerized tomography of the abdomen and

*The authors declare no conflict of interest.

${ }^{*}$ Corresponding author. pelvis confirmed the $18 \mathrm{~cm}$ pelvic mass with peritoneal and omental nodules and ascites. Inguinal lymphadenopathy measuring up to $4 \mathrm{~cm}$ was identified. Exploratory laparotomy identified right and left ovarian tumors and multiple tumor nodules on the peritoneum, omentum, and the surfaces of the uterus, bladder peritoneum, sigmoid colon and diaphragm were coated by thick tumor plaques. The patient underwent tumor debulking including omentectomy and bilateral salpingo-oophorectomy. However, optimal debulking could not be achieved and the uterus was not removable. Pathology examination revealed a bilateral $18 \times 12 \times 10 \mathrm{~cm}$ sized tumor with a smooth outer surface. On sectioning, the tumor showed solid and necrotic areas with thin-walled cystic spaces containing serous fluid. Microscopically, the tumor was composed of two different components, the larger of which was characterized by a monotonous population of epithelioid cells arranged predominantly in solid cords and nests (Figure 1). Coagulative tumor cell necrosis was frequently observed. On higher magnification, the tumor cells had narrow rims of amphophilic cytoplasm and enlarged round to oval nuclei with stippled evenly distributed chromatin, and inconspicuous to small nucleoli. Numerous mitotic figures were identified (15 - 20 


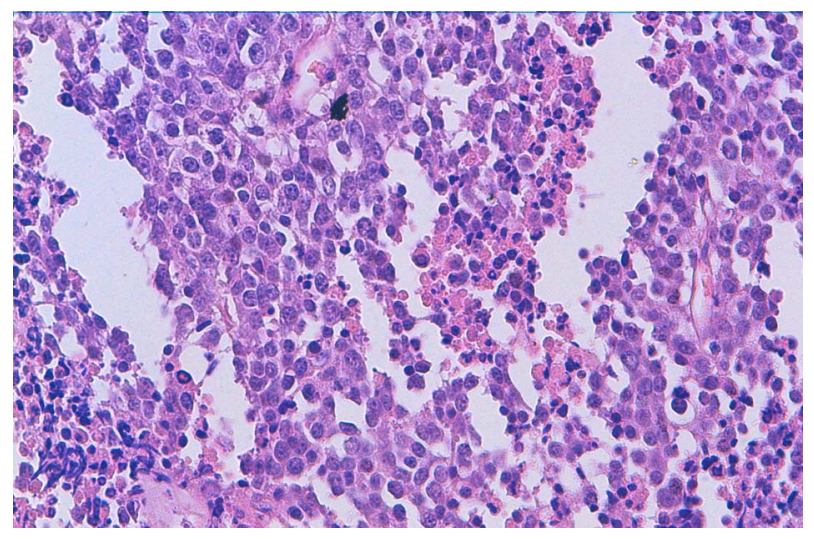

Figure 1. HES $\times$ 10: Tumor proliferation arranged in diffuse sheets representing the contingent of neuroendocrine carcinoma.

mitoses/10 HPF). The smaller component, which represented $10 \%-15 \%$ of the tumor, consisted of papillary formations (Figure 2) and glands lined by monotonous epithelial cells with enlarged nuclei. These features were characteristic of the histological appearance of highgrade serous carcinoma. Immunohistochemical studies for keratin 7 and neuroendocrine markers (chromogranin, synaptophysin, and CD56) showed that the larger component of the tumor was positive for neuroendocrine markers (Figure 3) and negative for keratin 7, and while the serous carcinoma component was diffusely positive for keratin 7 (Figure 4), but mostly negative for the neuroendocrine markers. Immunohistochemical stains for estrogen receptor (ER), progesterone receptor (PR), alphainhibin, calretinin, and thyroid transcription factor- 1 (TTF-1) were negative in both components. The morphological and immunohistochemical features of the larger component were compatible with those of neuroendocrine carcinoma of the non-small cell type. Therefore, a diagnosis of neuroendocrine carcinoma, non-small cell type, associated with serous carcinoma was made. The uterine serosa was involved by neuroendocrine carcinoma cells. Metastatic serous carcinoma components were not observed. Multiple tumor vascular emboli were identified in the uterine serosa and they were solely composed of neuroendocrine carcinoma cells. Following surgery, the patient received eight cycles of Taxol and Carboplatin. After a follow-up 8 months, the patient shows no evidence of disease.

\section{Discussion}

The case reported herein is the third case of neuroendocrine carcinoma, non-small cell type, associated with serous carcinoma. Although recently listed in the WHO Classification of Tumors as a primary ovarian tumor, LCNEC of the ovary is an exceedingly rare neoplasm, and only twenty-nine cases have been reported in the



Figure 2. HES × 10: Adenocarcinomatous proliferation of tubular and papillary architecture representing the papillary serous adenocarcinoma.

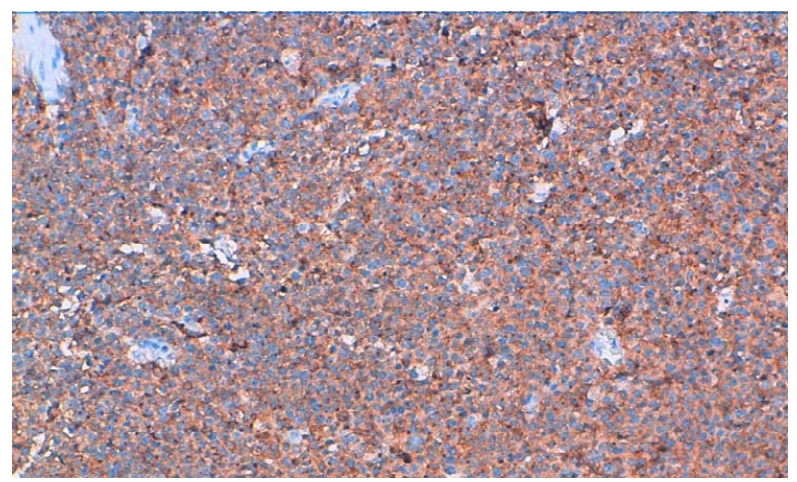

Figure 3. Granular cytoplasmic staining with anti chromogranin.

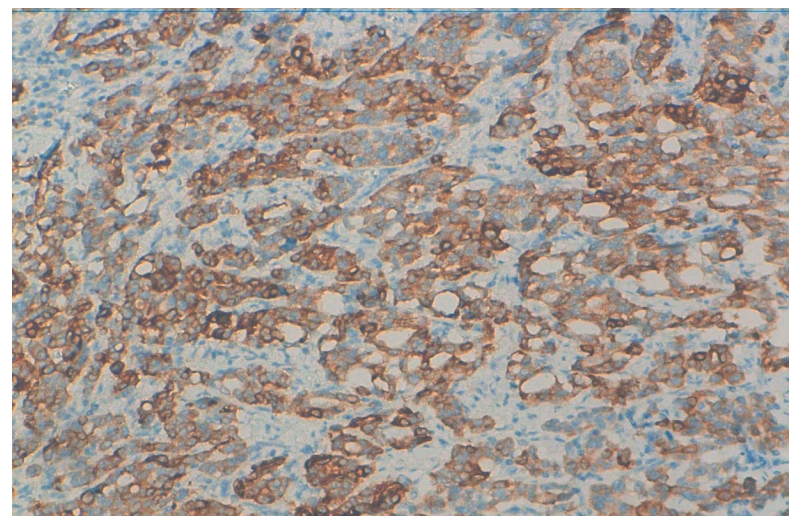

Figure 4. Tumor cells express the anti CK7.

literature so far [3]. Except for three cases of pure neuroendocrine carcinoma, all the remaining ones have been associated with ovarian epithelial tumors or germ cell tumors [4-6]. The only case of LCNEC admixed with low-grade serous carcinoma has recently been reported by Choi et al. [3]. Here we present another case of LCNEC associated with papillary serous carcinoma. The patients were in the reproductive age group or beyond (mean 56 years) and presented with symptoms related to 
a pelvic mass in the majority of cases. The histogenesis of ovarian neuroendocrine carcinomas remains unclear. Several theories have been proposed: 1) Neuroendocrine cells have been identified in normal epithelium from the female genital tract; 2) a primitive endodermal cell has been shown to be capable of differentiating into both endocrine and other cell types, 3) ovarian neuroendocrine neoplasms may develop from non-neuroendocrine cells in which there is activation of genes promoting neuroendocrine differentiation [3]. Diagnosis of LCNEC in routine pathology practice relies on typical morphology and adjunctive immunohistochemistry. The tumor is composed of medium to large neuroendocrine cells forming solid sheets and cords with abundant eosinophilic or granular cytoplasm. The nuclei show condensation of nuclear material (salt and pepper chromatin) and there are often large prominent nucleoli. Foci of necrosis and frequent mitoses are common findings. The neuroendocrine differentiation can be confirmed by immunohistochemistry that is positive for chromogranin, synaptophysin or other neuroendocrine markers [1]. The associated surface-epithelial components identified in the reported cases include mucinous borderline tumor, mucinous adenocarcinoma, endometrioid adenocarcinoma, mucinous adenoma/cystadenoma, adenocarcinoma NOS, admixed mucinous and endometrioid carcinoma, and serous carcinoma [7]. The LCNEC component in our case had morphology typical of neuroendocrine differentiation and an immunoprofile that was positive for neuroendocrine differentiation. However, the interesting feature of this case was that the intermingled ovarian epithelial tumor consisted of serous carcinoma. The high grade nuclear atypia, papillary configuration and glandular formation, and the psammoma bodies formed the classic morphology of a serous carcinoma. The reversed immunoprofile positive for epithelial markers and negative for neuroendocrine differentiation helped establish the diagnosis. Focal mucin secretion has been described in serous carcinomas and its prognostic significance is not established [8]. Whether it modifies their clinical behavior remains to be determined. The differential diagnosis of ovarian LCNEC is other primary and secondary neuroendocrine tumors. Small cell carcinoma has smaller cell size with molding and significant necrosis. Metastatic neuroendocrine carcinoma would almost never admix with ovarian surface epithelial tumors. Most of the reported cases presented with stage I disease, with tumors confined to one ovary [9]. In our case, the tumor was identified in both ovaries with extensive peritoneal disease. It is possible that the tumor started on one side and spread to the other ovary and the peritoneum. However, this assumption cannot be proven in a patient with such widespread disease. LCNEC of the ovary appears to be highly aggressive. The presence of LCNEC component in an otherwise usual type epithelial tumor should be reported because of the potential negative prognostic impact of this histologic finding, although there are too few cases in the literature to determine the prognostic impact of the percentage of LCNEC component in an epithelial ovarian tumor. Prior to our report, only the neuroendocrine component of the carcinoma has been described in the metastatic sites when metastasis occurs [7]. Like the previously reported cases, our patient's peritoneal metastases contained only neuroendocrine component. LCNEC and surface-epithelial components identical to those in the primary tumor. Most patients died of disease within 1 year of diagnosis despite extensive surgery and adjuvant chemotherapy [3, $4,8,9]$. Our patient presented with widespread peritoneal disease and showed response to the chemotherapy. Some authors have recently reported that the pattern of microsatellite instability (MSI) findings is a useful tool in determining whether a patient has double primary tumors or a single clonal tumor [10]. When MSI analysis was separately performed in the neuroendocrine and serous carcinoma components, the patterns for the MSI were quite different for all five markers.

We report the first case in Moroccan literature of an ovarian neuroendocrine carcinoma, non-small cell type, associated with a serous carcinoma. Using immunohistochemistry analysis a different origin for each tumor was suggested. When neuroendocrine carcinoma is presented in ovary, careful examination to search a surface epithelial tumor or teratoma is very important.

\section{REFERENCES}

[1] L. M. Roth, A. Tsubara, M. Dietel and H. Senzaki, "Miscellaneous Tumors and Tumor-Like Conditions of the Ovary,” In: F. A. Tavassoli and P. Devilee, Eds., Pathology and Genetics of Tumors of the Breast and Female Genital Organs, World Health Organization Classification of Tumors, IARC Press, Lyon, 2003, pp. 182-190.

[2] R. J. Collins, A. Cheung, H. Y. Ngan, L. C. Wong, S. Y. Chan and H. K. Ma, "Primary Mixed Neuroendocrine and Mucinous Carcinoma of the Ovary," Archives of Gynecology and Obstetrics, Vol. 248, No. 3, 1991, pp. 139143. doi:10.1007/BF02390091

[3] Y. D. Choi, J. S. Lee, C. Choi, C. S. Park and J. H. Nam, "Ovarian Neuroendocrine Carcinoma, Non-Small Cell Type, Associated with Serous Carcinoma,” Gynecologic Oncology, Vol. 104, No. 3, 2007, pp. 747-752. doi:10.1016/j.ygyno.2006.11.008

[4] K. Behnam, D. Kabus and M. Behnam, "Primary Ovarian Undifferentiated Non-Small Cell Carcinoma, Neuroendocrine Type,” Gynecologic Oncology, Vol. 92, No. 1, 2004, pp. 372-375. doi:10.1016/j.ygyno.2003.09.031

[5] P. Dundr, D. Fischerova, C. Povysil and D. Cibula, "Primary Pure Large-Cell Neuroendocrine Carcinoma of the Ovary," Pathology_Research and Practice, Vol. 204, No. 2, 2008, pp. 133-137. doi:10.1016/j.prp.2007.09.004 
[6] C. F. Lindboe, "Large Cell Neuroendocrine Carcinoma of the Ovary,” APMIS, Vol. 115, No. 2, 2007, pp. 169-176. doi:10.1111/j.1600-0463.2007.apm_570.x

[7] K. T. Chen, "Composite Large-Cell Neuroendocrine Carcinoma and Surface Epithelial-Stromal Neoplasm of the Ovary,” International Journal of Surgical Pathology, Vol. 8, No. 2, 2000, pp. 169-174. doi:10.1177/106689690000800214

[8] J. H. Eichhorn, W. D. Lawrence, R. H. Young and R. E. Scully, "Ovarian Neuroendocrine Carcinomas of NonSmall Cell Type Associated with Surface Epithelial Adenocarcinomas. A Study of Five Cases and Review of the Literature," International Journal of Gynecological Pa- thology, Vol. 15, 1996, pp. 303-314. doi:10.1097/00004347-199610000-00002

[9] R. J. Collins, A. Cheung, H. Y. Ngan, L. C. Wong, S. Y. Chan and H. K. Ma, "Primary Mixed Neuroendocrine and Mucinous Carcinoma of the Ovary," Archives of Gynecology and Obstetrics, Vol. 248, No. 3, 1991, pp. 139143. doi:10.1007/BF02390091

[10] M. Tang, Y. Pires, M. Schultz, I. Duarte, M. Gallegos, I. I. Wistuba, "Microsatellite Analysis of Synchronous and Metachronous Tumors: A Tool for Double Primary Tumor and Metastasis Assessment," Diagnostic Molecular Pathology, Vol. 12, No. 3, 2003, pp. 151-159. doi:10.1097/00019606-200309000-00006 